\title{
The Melatonin Receptor MT2 Is Partly Involved in the Anti-Inflammatory Effects of Melatonin in Rats With Type 2 Diabetes Mellitus
}

Hande Yapislar ( $\sim$ handeyapislar@gmail.com )

Acibadem Mehmet Ali Aydinlar University: Acibadem Universitesi https://orcid.org/0000-0002-2762357X

\section{Ebru Haciosmanoglu}

Bezmialem Vakif University: Bezmialem Vakif Universitesi

\section{Turkan Sarioglu}

Istanbul Kent University: Istanbul Kent Universitesi

\section{Sevgin Degirmencioglu}

Istanbul Bilim University Medical Faculty: Istanbul Bilim Universitesi Tip Fakultesi

Ibrahim Sogut

Istanbul Bilim University Medical Faculty: Istanbul Bilim Universitesi Tip Fakultesi

\section{Michael Poteser}

Medical University of Vienna: Medizinische Universitat Wien

\section{Cem Ekmekcioglu}

University of Vienna: Universitat Wien

\section{Research Article}

Keywords: insulin resistance, type 2 diabetes mellitus, melatonin

Posted Date: March 31st, 2021

DOI: https://doi.org/10.21203/rs.3.rs-307990/v1

License: (a) (1) This work is licensed under a Creative Commons Attribution 4.0 International License. Read Full License 


\section{THE MELATONIN RECEPTOR MT2 IS PARTLY INVOLVED IN THE ANTI- INFLAMMATORY EFFECTS OF MELATONIN IN RATS WITH TYPE 2 DIABETES MELLITUS}

Hande Yapislar $^{1 *}$, Ebru Haciosmanoglu ${ }^{2 \Delta}$, Turkan Sarioglu ${ }^{3 \Delta}$, Sevgin Degirmencioglu ${ }^{4}$, Ibrahim Sogut ${ }^{5}$, Michael Poteser ${ }^{6}$, Cem Ekmekcioglu $^{6}$

1 Department of Physiology, Faculty of Medicine, Acibadem Mehmet Ali Aydinlar University, Istanbul, Turkey

${ }^{2}$ Faculty of Medicine, Department of Biophysics, Bezmialem Vakif University, Istanbul, Turkey

${ }^{3}$ Department of Histology and Embryology, Fundamental Sciences, Faculty of Dentistry, Istanbul Kent University Istanbul, Turkey

${ }^{4}$ Vocational School of Health Services, Demiroglu Bilim University, Istanbul, Turkey.

5 Department of Biochemistry, Faculty of Medicine, Demiroglu Bilim University, Istanbul/Turkey

${ }^{6}$ Department of Environmental Health, Center for Public Health, Medical University of Vienna, 1090 Vienna, Austria

${ }^{\Delta}$ These authors contributed equally to this work

*Correspondence: Hande Yapislar, Department of Physiology, Faculty of Medicine, Acibadem Mehmet Ali Aydinlar University, Istanbul, Turkey 


\begin{abstract}
Background: Insulin resistance is associated with a pro-inflammatory state increasing the risk for complications in patients with type 2 diabetes mellitus (T2DM). In addition to its chronobiotic effects, the pineal hormone melatonin is also known to exert anti-inflammatory and antioxidant effects. Furthermore, melatonin was also suggested to affect insulin secretion and melatonin levels were reported to be decreased in T2DM patients
\end{abstract}

Aims: The aim of this study was therefore to investigate the effect of melatonin on inflammation in diabetic rats and to study the possible involvement of the melatonin receptor, MT2.

Materials and Methods: Male Sprague Dawley rats were randomly divided into four experimental groups ( $\mathrm{n}=10$ per group):1) control, 2) streptozotocin/nicotinamid induced diabetes type 2 (T2DM), 3) T2DM treated with melatonin (500 $\mu \mathrm{g} / \mathrm{kg} / \mathrm{day})$, and 4) T2DM treated with melatonin $(500 \mu \mathrm{g} / \mathrm{kg} /$ day $)$ and the selective MT2 receptor antagonist luzindole $(0.25 \mathrm{~g} / \mathrm{kg} /$ day $)$. Blood samples were taken for biochemical parameters and various tissue samples (liver, adipose tissue, brain) were removed for immunohistochemistry (IHC), western blot (WB) and Q-PCR analyses, respectively.

Results: Melatonin significantly reduced increased blood levels of liver transaminases (AST, ALT) and blood urea nitrogen (BUN) in diabetic rats. Furthermore, in liver and adipose tissue of treated rats, melatonin administration resulted in considerable lower expression of the increased inflammatory markers IL- $1 \beta$, IL-6, TNF- $\alpha$ and NF- $\kappa \mathrm{B}$, as shown by reduction in RNA and protein levels. The MT2 receptor was only partly involved in the anti-inflammatory effects of melatonin.

Conclusions: Our results suggest that melatonin has relevant anti-inflammatory effects in diabetic rats. Further studies in humans could prove the potential clinical benefit of melatonin supplementation in patients with T2DM.

\title{
Introduction
}


The worldwide prevalence of diabetes mellitus (DM) is increasing at an alarming rate. According to data from the World Health Organization, diabetes will be the seventh leading cause of death in 2030 [1] . Among DM types, type 2 diabetes mellitus (T2DM) accounts for more than $90 \%$ of DM cases and is characterized by hyperglycemia, insulin resistance in target tissues along with several comorbidities, including obesity, cardiovascular risks, renal failure and retinopathy $[2,3]$.

Insulin resistance is associated with a pro-inflammatory state increasing the risk for complications in T2DM [3, 4]. In this regard, several studies in patients with T2DM have reported higher secretion rates of inflammatory mediators such as IL-1 $\beta$, IL-6, TNF- $\alpha$ and their connection to complications and beta cell disorders $[3,5]$. The major role of IL-1 $\beta$ and TNF- $\alpha$ in insulin resistance in particular has been shown repeatedly. TNF- $\alpha$ impairs insulin signaling and decreases expression of insulin receptor [6]. IL-1 $\beta$, a key mediator of the inflammatory response, is also adversely involved in blood glucose control and beta cell dysfunction [7]. For example, a previous study in patients with T2DM indicated that the blockade of the interleukin-1 receptor (IL-1Ra) improves glycemic control through enhanced beta-cell secretory function [8].

Melatonin (N-acetyl-5-methoxytryptamine) is a circulating hormone that is mainly synthesized and released by the pineal gland. Its secretion is coupled to a robust circadian rhythm with the highest blood levels observed at night between approximately 2 and 4 a.m [9]. In addition to its chronobiotic and sleep inducing properties, melatonin is also well known for its antioxidant and anti-inflammatory effects[10,11] and several studies in the scientific literature have demonstrated the anti-inflammatory effects of melatonin in different disease models and conditions [12-15]. In this regard, both clinical and experimental data have provided evidence that melatonin reduces the secretion of pro-inflammatory cytokines and adhesion molecules and modulates inflammatory parameters[10]. In addition, it was shown that melatonin exerted protective effects on inflammation related to aging [16] and also alcoholic injury in the liver [17]. Furthermore, melatonin reduced inflammation in the central nervous system, which is suggested to be related to several neurological diseases[10], and also exerted analgesic effect in inflammatory pain conditions [18].

In addition to its anti-inflammatory effects, melatonin was also found to be associated with insulin secretion and DM. In this regard, studies suggest a direct link between insulin secretion from beta cells, glucose levels and melatonin secretion $[19,20]$. For example, a 
negative correlation between the nocturnal melatonin peak and insulin drop levels in serum was described [21, 22]. Also, in a study by Peschke et al., it was reported that patients with type 2 diabetes show lower circulating melatonin levels and higher insulin levels, respectively, with a statistical significant inverse correlation between these two hormones[23].

Melatonin mainly exerts its effects after binding to two melatonin receptors (MT), MT1 and MT2, respectively [24]. Both show different expression ratios in various tissues [24, 25]. Previous studies suggest that the immunomodulatory functions of melatonin are at least partly mediated after binding to the MT2 receptor [24-28].

Since there are currently only sparse data available regarding the association between melatonin, inflammation and diabetes we aimed to investigate the effect of melatonin on constitutively enhanced inflammation in diabetic rats and to also study the possible involvement of MT2 receptor. Another aim was to analyze the liver and metabolic blood profiles of melatonin treated rats, since previous studies showed beneficial effects of melatonin in rodents with diabetes or metabolic challenges [29-33] .

\section{Materials and Methods}

\section{Animals and Experimental Protocol}

All experiments on animals were approved by the Ethical Committee for Animal Experimentation of Acibadem Mehmet Ali Aydinlar University, Istanbul,Turkey. Male Sprague Dawley rats were purchased from TUBITAK MAM and were used for the experiments. After rats reached $200 \mathrm{~g}$ body weight T2DM was induced by intraperitoneal (i.p) injection of $100 \mathrm{mg} / \mathrm{kg}$ NAD (nicotinamide adenine dinucleotide) and $50 \mathrm{mg} / \mathrm{kg} \mathrm{STZ}$ (streptozotocin) respectively. STZ was applied 15 minutes after NAD administration. 48 hours later blood glucose levels were measured by a glucometer and rats with glucose levels of $\geq 200 \mathrm{mg} / \mathrm{kg}$ were included in the study.

Rats were randomly allocated to the following four experimental groups ( $\mathrm{n}=10$ per group):1) control, 2) streptozotozin/nicotiamide treated (T2DM), 3) T2DM treated with melatonin (500 $\mu \mathrm{g} / \mathrm{kg} / \mathrm{day})$, and 4) T2DM treated with melatonin $(500 \mu \mathrm{g} / \mathrm{kg} /$ day $)$ and the selective MT2 receptor antagonist luzindole $(0.25 \mathrm{~g} / \mathrm{kg} / \mathrm{day})$. Melatonin and luzindole treatments were started 48 hours after induction of diabetes with NAD+STZ. Both melatonin and luzindole were administered by daily i.p. injections during 6 weeks. During the treatment body weight was measured once a week. All animals survived during the experiment and prosperity of 
none of them has decreased according to the ethical welfare. At the end of the 6 weeks, animals were fasted overnight, anaesthetized using Ketamin/Rompun (50/10 mg/kg i.p), and sacrificed by cervical dislocation. Blood samples $(5 \mathrm{ml})$ were taken by their jugular veins and collected in tubes containing heparin to subsequently analyze biochemical parameters. Tissue samples were removed and weighed. One half of the tissue was immersed in paraformaldehyde solution for immunohistochemistry (IHC) applications and the other half was frozen quickly in liquid nitrogen and stored at $-80^{\circ} \mathrm{C}$ for western blot (WB) and Q-PCR analyses.

\section{Measurement of Biochemical Parameters}

Blood samples were centrifuged at $2500 \mathrm{x}$ g for $10 \mathrm{~min}$ at $4{ }^{\circ} \mathrm{C}$ and serum was separated afterwards. Serum alanine and aspartate aminotransferases (AST, ALT) and blood urea nitrogen (BUN),triglyceride, cholesterol and very low density lipoprotein (VLDL) levels were analyzed in serum on a Roche-HITACHI Cobas c311 auto analyzer (Roche Molecular Systems, Branchburg, NJ) by using commercial Roche kits.

\section{Immunohistochemistry (IHC) Protocol}

For histopathologic evaluation, routine paraffin wax embedding procedures were applied (LIT). Following fixation, tissues were dehydrated in graded ethanol series, clarification process was completed in xylene and slides embedded in parafin. 5- $\mu \mathrm{m}$-thick slices were cut via microtome (Leica RM2235). Formalin-fixed and paraffin-embedded tissue samples were further processed for evaluation of the severity of tissue inflammation.

After incubation in $56^{\circ} \mathrm{C}$ for 12 hours, sections were deparaffinizated in xylene and the rehydration process was applied through a descending series of alcohol to water. Antigen retrieval was performed by incubation of the sample in $10 \%$ citrate buffer $(\mathrm{pH} 6.0)$ at $250^{\circ} \mathrm{C}$ for $6 \mathrm{~min}$, with subsequent cooling to room temperature for $30 \mathrm{~min}$. Then, tissue sections on slides were marked with hydrophobic pen and rinsed in phosphate-buffered saline (PBS) with $5 \%$ Tween. For protein blocking and non-specific binding, the sections were incubated in 3\% $\mathrm{H} 2 \mathrm{O} 2$ for $20 \mathrm{~min}$ in dark. After rinsing in phosphate-buffered saline (PBS) the anti-polyvalent HRP kit (Invitrogen,USA) was used for the following steps. To reduce non-specific staining, sections were pretreated with the blocking solution for $20 \mathrm{~min}$ in a humidity chamber. After removing the blocking solution, slides were covered with primary antibodies used against IL- 
$1 \beta$ (Santa Cruz, USA), IL-6 (Abnova, Taiwan), TNF- $\alpha$ (Novus, USA) and NF- $\kappa$ B (SantaCruz, USA). Primary antibodies were directly applied on the sections and the slides were incubated overnight at $4^{\circ} \mathrm{C}$ in a humidified chamber. The negative control was incubated with a blocking solution without the primary antibody. After washing $(3 \times 5 \mathrm{~min})$ in PBS-Tween, sections were incubated with HRP-Streptovidin for $20 \mathrm{~min}$ in a humidified chamber and washed with PBS-Tween $(3 \times 5 \mathrm{~min})$. Freshly prepared AEC (Aminoethyl CarbazoleInvitrogen,USA) was applied as a chromogen for 8-15 minutes at room temperature. After the reaction was stopped by washing with deionized water, sections were counterstained with hematoxylin and washed under dripping water for 10-15 minutes for developing purple color. Finally, sections were covered with fixative, aqueous mounting solution (Bio-optica) and the stained sections were examined for IL-1 $\beta$, IL-6, TNF- $\alpha$, and NF- $\kappa$ B with BX53 Olympus Camera (DP72 Olympus Software)

\section{Protein Extraction Protocol From Tissues}

Tissue samples were homogenized with a homogenizer in RIPA buffer containing a protease inhibitor cocktail (Santa Cruz- sc-24948,USA). Homogenates were centrifuged at $13.000 \mathrm{rpm}$ for 10 minutes, and supernatants were obtained and kept in $-80{ }^{\circ} \mathrm{C}$ until subsequent analyses. Protein concentrations were determined by using a Quant-iT Protein Assay Kit (Invitrogen, USA).

\section{Western Blot Protocol}

Western blotting was performed according to a standard protocol [34, 35]. Equal amounts of protein (40 $\mu \mathrm{g} /$ well) were subjected to SDS-PAGE (\% 12 gels, Biorad,USA) and transferred to nitrocellulose membranes. After blocking in TBST (Tris-buffered saline, 0.1\% Tween 20) with 5\% BSA (Bovine Serum Albumin-Invitrogen,USA), membranes were probed overnight at $4{ }^{\circ} \mathrm{C}$ with the corresponding primary antibodies, e.g. anti-IL-1 $\beta$ (1:500; Abcam,UK); antiIL-6 (1:750; Novus,USA); anti-TNF- $\alpha,(1: 1000 ; \quad$ Invitrogen,USA) ; NF- $\kappa B,(1: 1000$; Invitrogen,USA). Anti-actin antibody (1:1000, Santa Cruz,USA) was used for the loading control. After the washing procedure, membranes were incubated with alkaline phosphatase conjugated secondary antibodies, IgG (1:5000 Santa Cruz, USA) for 1 hour at room temperature. The immunoreactive bands were visualized by a colorimetric detection kit 
(NBT-BCIP; ThermoFisher, USA) and protein amounts were analysed with ImageJ programme (1.46r, NIH USA).

\section{Real Time PCR (quantitative-q-PCR)}

For quantification of mRNA expression in tissues total RNAs were isolated by using the RNAzol RT solution (MRC, Canada) according to manufacturer's instructions. After completion of RNA isolation, RNA concentration and purity were calculated with NanoDrop 2000 (Thermo Scientific, USA). For this purpose $1 \mu 1$ RNA samples were pipetted in the device for determination of 260/280 and 260/230 ratios. Concentrations of all RNA samples were equalized before reverse transcription. RNAs were reverse transcribed into cDNA by using Script cDNA Synthesis Kit (Jena Bioscience, Germany). The resulting cDNA was amplified by qRT-PCR by using qPCR GreenMaster with the UNG Kit (Jena Bioscience, Germany). The real time conditions were carried out on the CFX-96 Real Time PCR System (Bio-Rad, USA) as follows: $50^{\circ} \mathrm{C}, 2 \mathrm{~min} ; 95^{\circ} \mathrm{C}, 2 \mathrm{~min}$; followed by 35 cycles of $95^{\circ} \mathrm{C}, 15 \mathrm{~s}$; $56{ }^{\circ} \mathrm{C}, 20 \mathrm{~s}$; and $72{ }^{\circ} \mathrm{C}, 30 \mathrm{~s}$. Relative mRNA transcripts levels were calculated according to the delta $\mathrm{CT}$ method $(2-\Delta \Delta \mathrm{CT})$ and, the relative expression of each gene was normalized to that of GAPDH. Primers were obtained from LGC Biosearch Technologies (Denmark). All measurements were performed in triplicate and specifity of amplicons was verified by Melting curve analysis. The specific primers were used including:

1. TNF- $\alpha$, forward: 5'-GCAGATGGGCTGTACCTTATC-3', reverse:5'GAAATGGCAAATCGGCTGAC-3';

2. IL-6, forward: 5'- GTCTTCTGGAGTTCCGTTTCT-3', reverse:5'GGGTTTCAGTATTGCTCTGAATG-3',

3. IL-1, forward: 5'- GGAAATGTTCCTCGTCCTAAGT-3', reverse: 5'ACTAGGCTTTGCTCTTCTCTTAC-3',

4. NF-кB, forward: 5'-GGTTACGGGAGATGTGAAGATG-3', reverse: 5'GTGGATGATGGCTAAGTGTAGG-3',

5. UbiquitinC, forward: 5'- TGATCTTTGCAGGCAAGCAG-3', reverse: 5'GGTGGACTCCTTCTGGATGT-3',

6. Hypoxanthine phosphoribosyltransferase(Hprt), forward: 5'GACCTCTCGAAGTGTTGGATAC-3', reverse: 


\section{Statistical Analyses}

Statistical analyses were performed using the GraphPad Prism (GraphPad Software, La Jolla, CA, USA). After checking for normal distribution an analysis of variance (ANOVA) test using the post-hoc Tukey test was applied. Results were expressed as the mean \pm SD anda $p$ value of $<0.05$ was considered as significant.

\section{Results}

\section{Biochemical Parameters}

AST, ALT, and BUN levels were significantly higher in the plasma of diabetic rats as compared to control group levels (Figure1 A-C). However, administration of melatonin to diabetic rats was associated with significantly lower levels of AST, ALT (both $\mathrm{p}<0.05$ compared to diabetic rats), and BUN ( $\mathrm{p}<0.01$ ). The MT2-receptor antagonist luzindole did not attenuate the beneficial effects of melatonin $(p>0.05)$.

Cholesterol $(\mathrm{p}<0.001)$, triglycerides $(\mathrm{p}<0.01)$ and VLDL $(\mathrm{p}<0.01)$ levels were significantly higher in the DM group and melatonin treatment significantly reduced these parameters in diabetic rats $(\mathrm{p}<0.01) ;(\mathrm{p}<0.05)$ and $(\mathrm{p}<0.05)$ respectively. (Data not shown). -The addition of luzindole to melatonin treated diabetic rats did not significantly influence the results on the various parameters assuming that the MT2 receptor is not mechanistically involved in the effects of melatonin on these parameters.

\section{Q-PCR}

Gene expression levels of the inflammatory cytokines IL-1 $\beta$, IL-6, TNF- $\alpha$ and NF- $\kappa$ B were analyzed in liver-, adipose- and brain tissues. In the liver tissue, relative expression of all four inflammatory parameters increased dramatically in the DM group $(\mathrm{p}<0.001)$ with melatonin treatment causing a remarkable significant decrease of all 4 inflammatory markers (IL-1 $\beta$, IL$6, \mathrm{NF}-\kappa \mathrm{B}=\mathrm{p}<0.001$ compared to the diabetic only group; TNF- $\alpha=\mathrm{p}<0.01$ ) (Figure2). The addition of luzindole to the MEL+DM group partly abolished the positive effects of melatonin on all cytokine levels $(\mathrm{p}<0.01$, Figure 2$)$ 
In adipose tissue, all cytokine gene expression levels increased significantly in the DM group compared to control rats $(\mathrm{p}<0.05)$ and melatonin treatment reduced the cytokine gene expression levels in all melatonin treated DM groups with a significance level of $\mathrm{p}<0.01$ for IL-1 $\beta$ and TNF- $\alpha$, and $p<0.001$ for IL- 6 and NF- $\kappa B$, respectively, compared to the untreated DM group (Figure3). Luzindole treatment increased IL-1 $\beta$, TNF- $\alpha$ and NF- $\kappa$ B gene expression levels significantly compared to MEL treated DM groups $(p<0.01, p<0.001$, $\mathrm{p}<0.05$ respectively). Mean IL-6 levels were not significantly different in the LUZ administered group $(\mathrm{p}>0.05)$

In the brain tissue of DM rats IL-1 $\beta$ and IL-6 cytokine levels were significantly higher compared to controls $(\mathrm{p}<0.05)$ with melatonin treatment reducing the expression of these cytokines to approximately control levels $(\mathrm{p}<0.05)$ (Figure4). No significant intergroup differences between DM and DM+MEL were observed among the expression levels of the other two cytokines genes $(\mathrm{p}>0.05)$. Furthermore, luzindole treatment did not affect the beneficial effects of melatonin regarding IL-1 $\beta$ and IL-6.

\section{Western Blot}

IL-1 $\beta$, IL-6,TNF- $\alpha$ and NF- $\kappa B$ protein levels were studied by western blotting in liver, and brain tissues. $\beta$-actin was used as the reference protein and the cytokine band absorbance level to $\beta$-actin's level in each well was compared. Densitometric analyses were perfomed my ImageJ Analyze Programme (1.46r, NIH USA)

In the liver tissue of DM rats IL- $1 \beta$ and TNF- $\alpha$ protein levels were significantly higher than in the liver of the control animals $(\mathrm{p}<0.01, \mathrm{p}<0.001$ respectively, Figure 5). The addition of melatonin to diabetic rats significantly reduced the levels of IL-1 $\beta(p<0.01)$, TNF- $\alpha(p<0.001)$ and NF- $\mathrm{B} B(\mathrm{p}<0.01)$. The MT2-receptor appeared to be at least partly involved in the effects of melatonin, since protein levels of IL- $1 \beta$, and TNF- $\alpha$ were significantly higher in the DM+MEL+LUZ group compared to the DM+MEL group ( $\mathrm{p}<0.05$ for IL- $1 \beta ; \mathrm{p}<0.01$ for TNF- $\alpha$. IL-6 levels were not significantly different between the groups (Figure 5).

In the brain tissue all cytokine levels were significantly higher in the DM group $(\mathrm{p}<0.001$ for IL-1 $\beta$ and TNF- $\alpha ; p<0.01$ for IL-6 and NF- $\kappa B$, (Suppl. Figure) with all exhibiting significant lower values in DM+MEL compared to the DM group ( $p<0.001$ for IL-1 $\beta$ and TNF- $\alpha ; p<0.01$ 


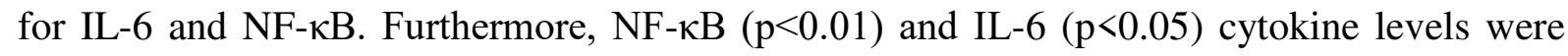
significantly higher in DM+MEL+LUZ group than in DM+MEL group.

\section{Immunohistochemistry}

Immunostainings of all cytokine levels (IL-1 $\beta$, IL-6 ,TNF- $\alpha$ and NF- $\kappa$ B) exhibit an intense immunoreactivity in DM group. A representative immunohistochemical result from all cytokine levels in the liver is presented in Figure 6 (A-D). Low level of cytokine immunoreactivity was observed in DM+MEL group. The DM+MEL+LUZ group exhibit moderate intensity of cytokine immunoreactivity. Immunohistochemistry results of cytokine expressions for brain tissue sections are demonstrated in a supplement figure .

\section{Discussion}

This study in particular showed that administration of melatonin significantly reduced inflammation in the liver and adipose tissue of diabetic rats with the positive effects being partly explained by the involvement of the MT2 receptor. In addition, some metabolic improvements were detected in melatonin treated diabetic animals.

Melatonin has been related to glucose and insulin homeostasis regulation in different ways. For example, pinealectomized rats show insulin resistance and glucose intolerance [36], and in Goto Kakizaki rats, a model for type 2 diabetes, nocturnal melatonin secretion was reduced whereas higher than normal levels of insulin reported . Higher levels of insulin are considered a hallmark of T2D [37]. Concordantly, also in T2DM patients reduced circulating melatonin and elevated insulin levels were found [38]. In addition, in T1DM melatonin levels were significantly lower when compared to the control group [39].

It is well known that inflammatory markers are elevated in insulin resistant T2DM patients [40-42] with an impaired balance between $\mathrm{T}$ cell subtypes might playing an important role in the deterioration of glucose homeostasis in both type 1 and type 2 diabetes [43]. Also, experimental studies in recent years have shown that interferon- $\gamma$ expression is increased in 
diabetic mice and induces adipose tissue inflammation [44]. Furthermore, c-Jun N- kinases (JNKs), which are key regulators of inflammation, exhibit a marked increase in obesity. In this regard an increased expression of TNF- $\alpha$, which is a potent regulator of JNKs, has been demonstrated in obese mice connecting a link between obesity and insulin resistance [45]. The JNK pathway is activated in several tissues in DM and inhibition of JNK is known to ameliorate insulin resistance [46]. Also, JNK knock down mice have a decreased expression of pro-inflammatory cytokines such as TNF- $\alpha$ or IL-6, which might protect against insulin resistance in T2DM [47].

All in all, the role of inflammation in DM has been widely investigated [48, 49]. On the other hand, there are only scarce data available regarding the relationship between melatonin, inflammation and type 2 diabetes and its complications. For example, in early studies with young ZDF (Zucker diabetic fatty) rats, an experimental model of the metabolic syndrome and type 2 diabetes, oral administration of melatonin reduced the levels of pro-inflammatory cytokines such as IL-6, TNF- $\alpha$, and also CRP, oxidative stress or low-grade inflammation [50]. Furthermore, Ozkanlar et al. reported that melatonin administration decreases IL-1 $\beta$ levels in the serum of rats with induced T1DM [51]. Moreover in T2DM patients with chronic peridontitis, melatonin supplementation decreased the levels of IL-6 in serum [52]. In addition, melatonin was shown to prevent the production of pro-inflammatory cytokines such as IL-1 $\beta$ and TNF- $\alpha$ in diabetic retinopathy [53].

Melatonin significantly reduced NF- $\kappa \mathrm{B}$ levels in our diabetic rats. NF- $\kappa \mathrm{B}$ is a transcription factor, which mediates the production of the pro-inflammatory cytokines TNF- $\alpha$, IL-1 $\beta$ and IL-6, also playing an important role in innate immunity. Previous studies indicated that melatonin inhibits the transcriptional activation of TNF- $\alpha$ and IL- $1 \beta[54,55]$ by blocking NF$\kappa \mathrm{B}$ binding to DNA [56]. Also, in ovarium tissue sections in ovarium injured diabetic rats [57] and in experimental diabetic neuropathy [58], NF- $\kappa \mathrm{B}$ immunoexpression was found to be significantly lower in melatonin-treated diabetic rats.

Melatonin primarily exerts its effects through two G-protein coupled membrane receptors (GPCRs), MT1 and MT2, respectively [24]. Although receptor distributions may vary, melatonin receptors are expressed in many different organs and tissues with the MT2 (Mel1b) receptor also being found in the endocrine pancreas [59], where melatonin has been shown to reduce the rate of insulin secretion after receptor activation [60]. MT receptors have also been identified in leukocytes [61-63] and melatonin is suggested to modulate (or regulate) the 
immune system by (for example) stimulating lymphocytes via these receptors, predominantly via the MT2 receptor [64]. Complete removal of the pineal gland causes partial deterioration in the immune responses of rats $[65,66]$, and administration of melatonin in pinealectomized animals reverses the negative effect on immune responses [66].

Luzindole is a selective melatonin receptor antagonist and has a high affinity for the MT2 receptor. It was, for example, observed that mice treated with luzindole produced lower levels of IgG than non-treated controls [64]. In our study we also aimed to investigate whether melatonin exerted its possible anti-inflammatory effects via the MT2 receptor. We demonstrated that some of the potent anti-inflammatory effects of melatonin were lost, at least tendentially, after application of luzindole. In accordance, for example, in mesenchymal stem cells exposed to inflammation, it was shown that melatonin reduced the amount of reactive oxygen species in these cells and acted as an anti-inflammatory agent; however, administration of luzindole reversed this effect [67]. In another study, it has been shown that melatonin reduces colonic and gastric inflammation, but administration of luzindole abolished these benefical effects of melatonin [68]. All these data, together with ours, demonstrate that MT2 receptors might have a role in anti-inflammatory effects of melatonin. Although the results of our study are in line with other studies examining the effects of melatonin and luzindole on inflammation, it is probably the first extensive study on diabetes, inflammation and melatonin in the literature.

\section{Conclusions, strengths and limitations}

All in all our findings are compatible with the few available data of former studies suggesting that administration of melatonin reduces inflammation in diabetes mellitus. The strengths of our study are the application of a variety of methods, including different tissues with four different inflammation markers, and also studying the potential involvement of the MT2 receptor in mediating the potential beneficial effects of melatonin. Nevertheless, our study has also limitations. We did not study the involvement of the MT1 receptor. However, although high affinity selective agents are available for the MT2 receptor, this does not apply to the MT1 receptor [69]. Nevertheless, recent data indicated that MT2 receptors have a more direct involvement in T2DM [70].

Also we did not adressed oxidative stress markers in our study. Oxidative stress has also been suggested to be involved in inflammation [71]. However, analysing oxidative stress was beyond the scope of our study. 


\section{Future perspectives}

Future clinical studies could adress the effect of melatonin supplementation in patients with diabetes mellitus, preferably with a higher grade of inflammation. It would also be interesting to include older diabetic patients, since it is known that aging is associated with a lower nocturnal melatonin secretion [72, 73], which could aggravate the inflammatory state. Additionally, aging is associated with a chronic low-grade pro-inflammatory state [74] suggesting a multiplying effect of low melatonin, diabetes and inflammation.

\section{Acknowledgement}

This study was supported financially by the Scientific and Technological Research Council of Turkey (TÜBİTAK; project number: 114S852)

\section{References}

1. Mathers, C.D. and D. Loncar, Projections of global mortality and burden of disease from 2002 to 2030. PLoS Med, 2006. 3(11): p. e442.

2. Gold, M.S. and J.t. Bordley, Pancreaticoduodenectomy for bleeding periampullary tumors. Arch Surg, 1990. 125(5): p. 675-7.

3. Scheen, A.J. and L.F. Van Gaal, Combating the dual burden: therapeutic targeting of common pathways in obesity and type 2 diabetes. Lancet Diabetes Endocrinol, 2014. 2(11): p. 911-22.

4. Mishra, M., et al., Level of serum IL-12 and its correlation with endothelial dysfunction, insulin resistance, proinflammatory cytokines and lipid profile in newly diagnosed type 2 diabetes. Diabetes Res Clin Pract, 2011. 94(2): p. 255-61.

5. Chen, W., E. Balland, and M.A. Cowley, Hypothalamic Insulin Resistance in Obesity: Effects on Glucose Homeostasis. Neuroendocrinology, 2017. 104(4): p. 364-381.

6. Peraldi, P. and B. Spiegelman, TNF-alpha and insulin resistance: summary and future prospects. Mol Cell Biochem, 1998. 182(1-2): p. 169-75.

7. Morris, D.L., Minireview: Emerging Concepts in Islet Macrophage Biology in Type 2 Diabetes. Mol Endocrinol, 2015. 29(7): p. 946-62.

8. Larsen, C.M., et al., Interleukin-1-receptor antagonist in type 2 diabetes mellitus. N Engl J Med, 2007. 356(15): p. 1517-26.

9. Grivas, T.B. and O.D. Savvidou, Melatonin the "light of night" in human biology and adolescent idiopathic scoliosis. Scoliosis, 2007. 2: p. 6.

10. Esposito, E. and S. Cuzzocrea, Antiinflammatory activity of melatonin in central nervous system. Curr Neuropharmacol, 2010. 8(3): p. 228-42.

11. Kostoglou-Athanassiou, I., Therapeutic applications of melatonin. Ther Adv Endocrinol Metab, 2013. 4(1): p. 13-24. 
12. MacDonald, I.J., et al., Reconsidering the Role of Melatonin in Rheumatoid Arthritis. Int J Mol Sci, 2020. 21(8).

13. Chen, Y., et al., Melatonin attenuated inflammatory reaction by inhibiting the activation of p38 and NFkappaB in taurocholateinduced acute pancreatitis. Mol Med Rep, 2018. 17(4): p. 5934-5939.

14. Farez, M.F., et al., Anti-inflammatory effects of melatonin in multiple sclerosis. Bioessays, 2016. 38(10): p. 1016-26.

15. Shang, B., et al., Protective effect of melatonin on myenteric neuron damage in experimental colitis in rats. Fundam Clin Pharmacol, 2016. 30(2): p. 117-27.

16. Cuesta, S., et al., Melatonin improves inflammation processes in liver of senescenceaccelerated prone male mice (SAMP8). Exp Gerontol, 2010. 45(12): p. 950-6.

17. Hu, S., et al., Melatonin protects against alcoholic liver injury by attenuating oxidative stress, inflammatory response, and apoptosis. Eur J Pharmacol, 2009. 616(1-3): p. 287-92.

18. Laste, G., et al., Melatonin administration reduces inflammatory pain in rats. J Pain Res, 2012. 5: p. 359-62.

19. Peschke, E., et al., Evidence for a melatonin receptor within pancreatic islets of neonate rats: functional, autoradiographic, and molecular investigations. J Pineal Res, 2000. 28(3): p. 15664.

20. Picinato, M.C., et al., Melatonin inhibits insulin secretion and decreases PKA levels without interfering with glucose metabolism in rat pancreatic islets. J Pineal Res, 2002. 33(3): p. 15660.

21. Rasmussen, D.D., et al., Daily melatonin administration at middle age suppresses male rat visceral fat, plasma leptin, and plasma insulin to youthful levels. Endocrinology, 1999. 140(2): p. 1009-12.

22. Wolden-Hanson, T., et al., Daily melatonin administration to middle-aged male rats suppresses body weight, intraabdominal adiposity, and plasma leptin and insulin independent of food intake and total body fat. Endocrinology, 2000. 141(2): p. 487-97.

23. Peschke, E., et al., Melatonin and type 2 diabetes - a possible link? J Pineal Res, 2007. 42(4): p. $350-8$.

24. Ekmekcioglu, C., Melatonin receptors in humans: biological role and clinical relevance. Biomed Pharmacother, 2006. 60(3): p. 97-108.

25. Ekmekcioglu, C., et al., The melatonin receptor subtype MT2 is present in the human cardiovascular system. J Pineal Res, 2003. 35(1): p. 40-4.

26. Tsai, T.H., et al., Melatonin attenuated the brain damage and cognitive impairment partially through MT2 melatonin receptor in mice with chronic cerebral hypoperfusion. Oncotarget, 2017. 8(43): p. 74320-74330.

27. Wongprayoon, P. and P. Govitrapong, Melatonin attenuates methamphetamine-induced neuroinflammation through the melatonin receptor in the SH-SY5Y cell line. Neurotoxicology, 2015. 50: p. 122-30.

28. Soderquist, F., P.M. Hellstrom, and J.L. Cunningham, Human gastroenteropancreatic expression of melatonin and its receptors MT1 and MT2. PLoS One, 2015. 10(3): p. e0120195.

29. Das, N., et al., Melatonin protects against lipid-induced mitochondrial dysfunction in hepatocytes and inhibits stellate cell activation during hepatic fibrosis in mice. J Pineal Res, 2017. 62(4).

30. Ou, T.H., et al., Melatonin Improves Fatty Liver Syndrome by Inhibiting the Lipogenesis Pathway in Hamsters with High-Fat Diet-Induced Hyperlipidemia. Nutrients, 2019. 11(4).

31. Hajam, Y.A. and S. Rai, Melatonin and insulin modulates the cellular biochemistry, histoarchitecture and receptor expression during hepatic injury in diabetic rats. Life Sci, 2019. 239: p. 117046.

32. Cheshchevik, V.T., et al., Corrections by melatonin of liver mitochondrial disorders under diabetes and acute intoxication in rats. Cell Biochem Funct, 2011. 29(6): p. 481-8. 
33. Baydas, G., H. Canatan, and A. Turkoglu, Comparative analysis of the protective effects of melatonin and vitamin E on streptozocin-induced diabetes mellitus. J Pineal Res, 2002. 32(4): p. 225-30.

34. Laemmli, U.K., Cleavage of structural proteins during the assembly of the head of bacteriophage T4. Nature, 1970. 227(5259): p. 680-5.

35. Koc, E.C., et al., Impaired mitochondrial protein synthesis in head and neck squamous cell carcinoma. Mitochondrion, 2015. 24: p. 113-21.

36. Zanquetta, M.M., et al., Calorie restriction reduces pinealectomy-induced insulin resistance by improving GLUT4 gene expression and its translocation to the plasma membrane. J Pineal Res, 2003. 35(3): p. 141-8.

37. Peschke, E., et al., Diabetic Goto Kakizaki rats as well as type 2 diabetic patients show a decreased diurnal serum melatonin level and an increased pancreatic melatonin-receptor status. J Pineal Res, 2006. 40(2): p. 135-43.

38. Peschke, E. and E. Muhlbauer, New evidence for a role of melatonin in glucose regulation. Best Pract Res Clin Endocrinol Metab, 2010. 24(5): p. 829-41.

39. Kor, Y., et al., Preliminary study: Evaluation of melatonin secretion in children and adolescents with type 1 diabetes mellitus. Indian J Endocrinol Metab, 2014. 18(4): p. 565-8.

40. Lontchi-Yimagou, E., et al., Diabetes mellitus and inflammation. Curr Diab Rep, 2013. 13(3): p. 435-44.

41. Karstoft, K. and B.K. Pedersen, Exercise and type 2 diabetes: focus on metabolism and inflammation. Immunol Cell Biol, 2016. 94(2): p. 146-50.

42. Amin, M.N., et al., How the association between obesity and inflammation may lead to insulin resistance and cancer. Diabetes Metab Syndr, 2019. 13(2): p. 1213-1224.

43. Zhen, Y., et al., Alterations of peripheral $C D 4+C D 25+F o x p 3+T$ regulatory cells in mice with STZ-induced diabetes. Cell Mol Immunol, 2012. 9(1): p. 75-85.

44. Zhang, $\mathrm{H}_{\text {., }}$ et al., Interferon-gamma induced adipose tissue inflammation is linked to endothelial dysfunction in type 2 diabetic mice. Basic Res Cardiol, 2011. 106(6): p. 1135-45.

45. Ventre, J., et al., Targeted disruption of the tumor necrosis factor-alpha gene: metabolic consequences in obese and nonobese mice. Diabetes, 1997. 46(9): p. 1526-31.

46. Kaneto, H., et al., Possible novel therapy for diabetes with cell-permeable JNK-inhibitory peptide. Nat Med, 2004. 10(10): p. 1128-32.

47. Tuncman, G., et al., Functional in vivo interactions between JNK1 and JNK2 isoforms in obesity and insulin resistance. Proc Natl Acad Sci U S A, 2006. 103(28): p. 10741-6.

48. Donath, M.Y., Multiple benefits of targeting inflammation in the treatment of type 2 diabetes. Diabetologia, 2016. 59(4): p. 679-82.

49. Burhans, M.S., et al., Contribution of Adipose Tissue Inflammation to the Development of Type 2 Diabetes Mellitus. Compr Physiol, 2018. 9(1): p. 1-58.

50. Agil, A., et al., Melatonin ameliorates low-grade inflammation and oxidative stress in young Zucker diabetic fatty rats. J Pineal Res, 2013. 54(4): p. 381-8.

51. Ozkanlar, S., et al., Melatonin Modulates the Immune System Response and Inflammation in Diabetic Rats Experimentally-Induced by Alloxan. Horm Metab Res, 2016. 48(2): p. 137-44.

52. Bazyar, H., et al., The effects of melatonin supplementation in adjunct with non-surgical periodontal therapy on periodontal status, serum melatonin and inflammatory markers in type 2 diabetes mellitus patients with chronic periodontitis: a double-blind, placebocontrolled trial. Inflammopharmacology, 2019. 27(1): p. 67-76.

53. Jiang, T., et al., Protective Effects of Melatonin on Retinal Inflammation and Oxidative Stress in Experimental Diabetic Retinopathy. Oxid Med Cell Longev, 2016. 2016: p. 3528274.

54. Li, J.H., et al., Melatonin reduces inflammatory injury through inhibiting NF-kappaB activation in rats with colitis. Mediators Inflamm, 2005. 2005(4): p. 185-93.

55. Sasaki, M., et al., Melatonin reduces TNF-a induced expression of MAdCAM-1 via inhibition of NF-kappaB. BMC Gastroenterol, 2002. 2: p. 9. 
56. Chuang, J.I., et al., Effect of melatonin on NF-kappa-B DNA-binding activity in the rat spleen. Cell Biol Int, 1996. 20(10): p. 687-92.

57. Nayki, U., et al., The effect of melatonin on oxidative stress and apoptosis in experimental diabetes mellitus-related ovarian injury. Gynecol Endocrinol, 2016. 32(5): p. 421-6.

58. Negi, G., A. Kumar, and S.S. Sharma, Melatonin modulates neuroinflammation and oxidative stress in experimental diabetic neuropathy: effects on NF-kappaB and Nrf2 cascades. J Pineal Res, 2011. 50(2): p. 124-31.

59. Zibolka, J., et al., Distribution and density of melatonin receptors in human main pancreatic islet cell types. J Pineal Res, 2018. 65(1): p. e12480.

60. Muhlbauer, E. and E. Peschke, Evidence for the expression of both the MT1- and in addition, the MT2-melatonin receptor, in the rat pancreas, islet and beta-cell. J Pineal Res, 2007. 42(1): p. 105-6.

61. Poppema, S., et al., Monoclonal antibodies (MT1, MT2, MB1, MB2, MB3) reactive with leukocyte subsets in paraffin-embedded tissue sections. Am J Pathol, 1987. 127(3): p. 418-29.

62. Dubocovich, M.L. and M. Markowska, Functional MT1 and MT2 melatonin receptors in mammals. Endocrine, 2005. 27(2): p. 101-10.

63. Glebezdina, N.S., et al., Molecular Mechanisms of Control of Differentiation of Regulatory TLymphocytes by Exogenous Melatonin. Dokl Biochem Biophys, 2019. 484(1): p. 13-16.

64. Drazen, D.L. and R.J. Nelson, Melatonin receptor subtype MT2 (Mel 1b) and not mt1 (Mel 1a) is associated with melatonin-induced enhancement of cell-mediated and humoral immunity. Neuroendocrinology, 2001. 74(3): p. 178-84.

65. Haldar, C., D. Haussler, and D. Gupta, Effect of the pineal gland on circadian rhythmicity of colony forming units for granulocytes and macrophages (CFU-GM) from rat bone marrow cell cultures. J Pineal Res, 1992. 12(2): p. 79-83.

66. del Gobbo, V., et al., Pinealectomy inhibits interleukin-2 production and natural killer activity in mice. Int J Immunopharmacol, 1989. 11(5): p. 567-73.

67. Liu, X., et al., Melatonin mediates protective effects on inflammatory response induced by interleukin-1 beta in human mesenchymal stem cells. J Pineal Res, 2013. 55(1): p. 14-25.

68. Cevik, H., et al., Exposure to continuous darkness ameliorates gastric and colonic inflammation in the rat: both receptor and non-receptor-mediated processes. J Gastroenterol Hepatol, 2005. 20(2): p. 294-303.

69. Descamps-Francois, C., et al., Design and synthesis of naphthalenic dimers as selective MT1 melatoninergic ligands. J Med Chem, 2003. 46(7): p. 1127-9.

70. Karamitri, A., et al., Type 2 diabetes-associated variants of the MT2 melatonin receptor affect distinct modes of signaling. Sci Signal, 2018. 11(545).

71. Dandekar, A., R. Mendez, and K. Zhang, Cross talk between ER stress, oxidative stress, and inflammation in health and disease. Methods Mol Biol, 2015. 1292: p. 205-14.

72. Hill, S.M., et al., Age-related decline in melatonin and its MT1 receptor are associated with decreased sensitivity to melatonin and enhanced mammary tumor growth. Curr Aging Sci, 2013. 6(1): p. 125-33.

73. Waldhauser, F., et al., Alterations in nocturnal serum melatonin levels in humans with growth and aging. J Clin Endocrinol Metab, 1988. 66(3): p. 648-52.

74. Bektas, A., et al., Aging, inflammation and the environment. Exp Gerontol, 2018. 105: p. 1018. 


\section{Figures}

A

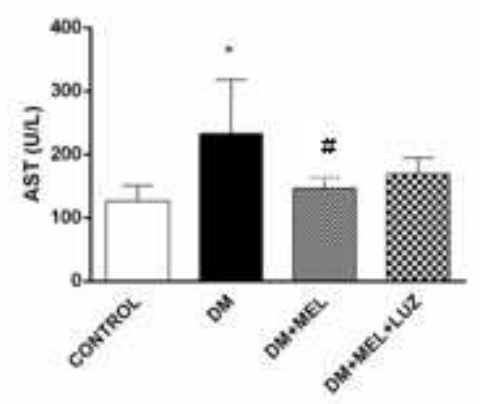

B

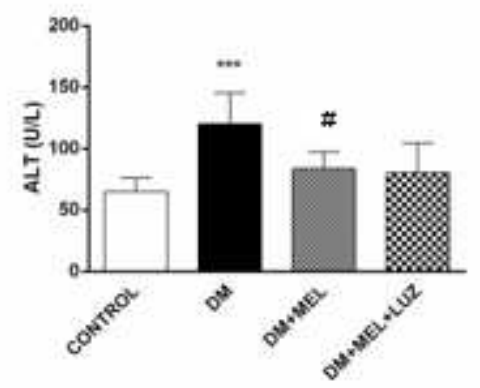

C

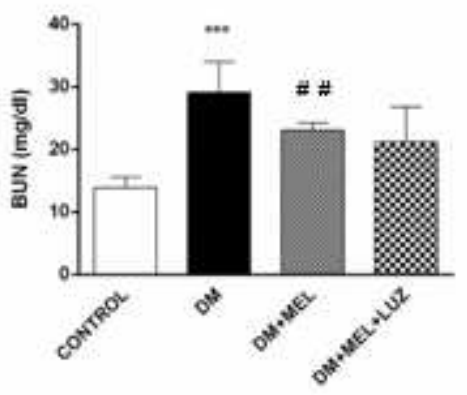

\section{Figure 1}

Biochemical analysis results of control and experimental groups. AST (A), ALT (B), BUN (C), levels in plasma from control, DM, DM plus treatment with melatonin $(500 \mu \mathrm{g} / \mathrm{kg} /$ day $), D M$ plus treatment with luzindole $(0.25 \mathrm{~g} / \mathrm{kg} /$ day $)$ and melatonin $(500 \mu \mathrm{g} / \mathrm{kg} /$ day). Values are represented as mean $\pm S D$.

Significant differences compared to controls $=$ * Significant differences compared to $D M=\# *$, $\#=p<0.05$ , **, \#\# = p<0.01, ***, \#\#\# = p<0.001 

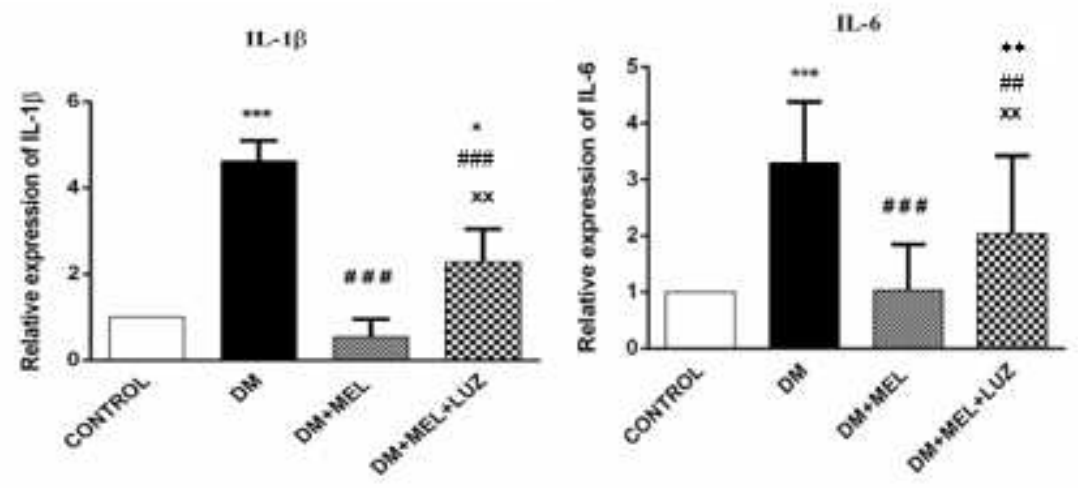

C

D
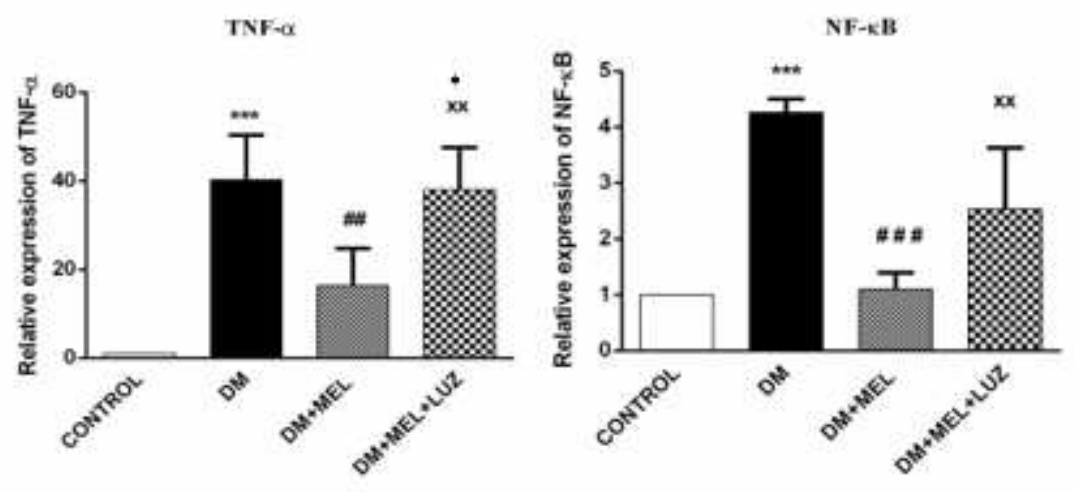

Figure 2

Q-PCR analysis results of cytokine levels in liver. IL-1 $\beta$ (A) , IL-6 (B), TNF-a (C), NF-KB (D) levels in liver tissues of control, DM, DM plus treatment with melatonin $(500 \mu \mathrm{g} / \mathrm{kg} /$ day), DM plus treatment with luzindole $(0.25 \mathrm{~g} / \mathrm{kg} /$ day $)$ and melatonin $(500 \mu \mathrm{g} / \mathrm{kg} /$ day $)$. Values are represented as mean $\pm S D$.

Significant differences compared to controls $=*$ Significant differences compared to $D M=$ \# Significant differences compared to $D M+M E L=x *, \#, x=p<0.05, * \star, \# \#, x x=p<0.01, \star \star \star, \# \# \#, x x x=p<0.001$ 

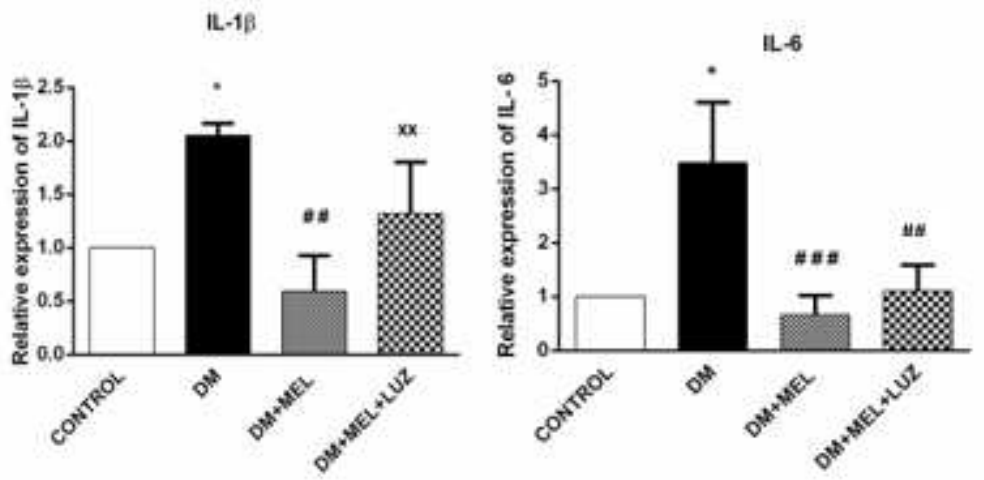

C

D
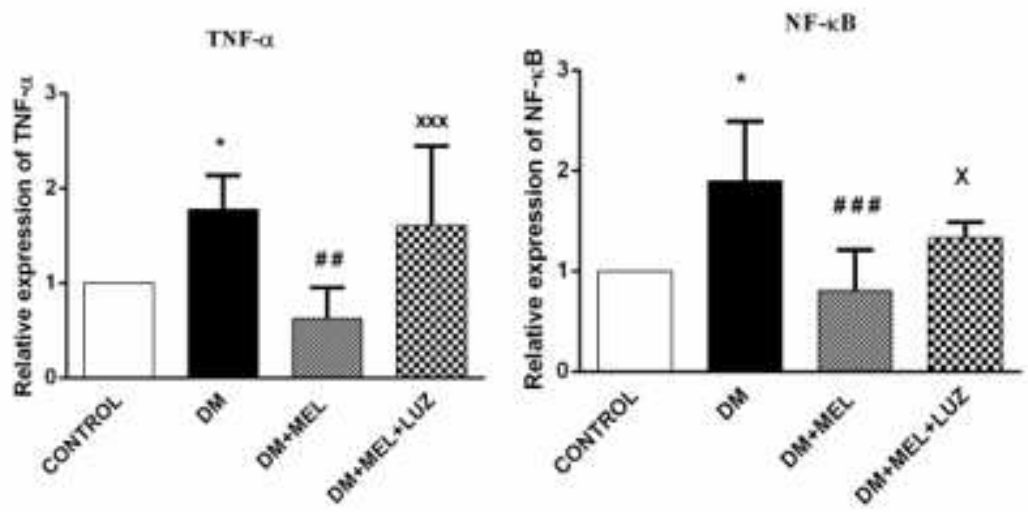

Figure 3

Q-PCR analysis results of cytokine levels in adipose tissue. IL-1 $\beta$ (A) , IL-6 (B), TNF-a (C), NF-KB (D) levels in adipose tissues of control, DM, DM plus treatment with melatonin $(500 \mu \mathrm{g} / \mathrm{kg} /$ day $), D M$ plus treatment with luzindole $(0.25 \mathrm{~g} / \mathrm{kg} /$ day) and melatonin $(500 \mu \mathrm{g} / \mathrm{kg} /$ day). Values are represented as mean $\pm S D$. Significant differences compared to controls $=$ * Significant differences compared to DM $=$ \# Significant differences compared to DM+MEL $=x *, \#, x=p<0.05, \star \star, \# \#, x x=p<0.01, \star \star \star, \# \# \#, x x x=p<0.001$ 

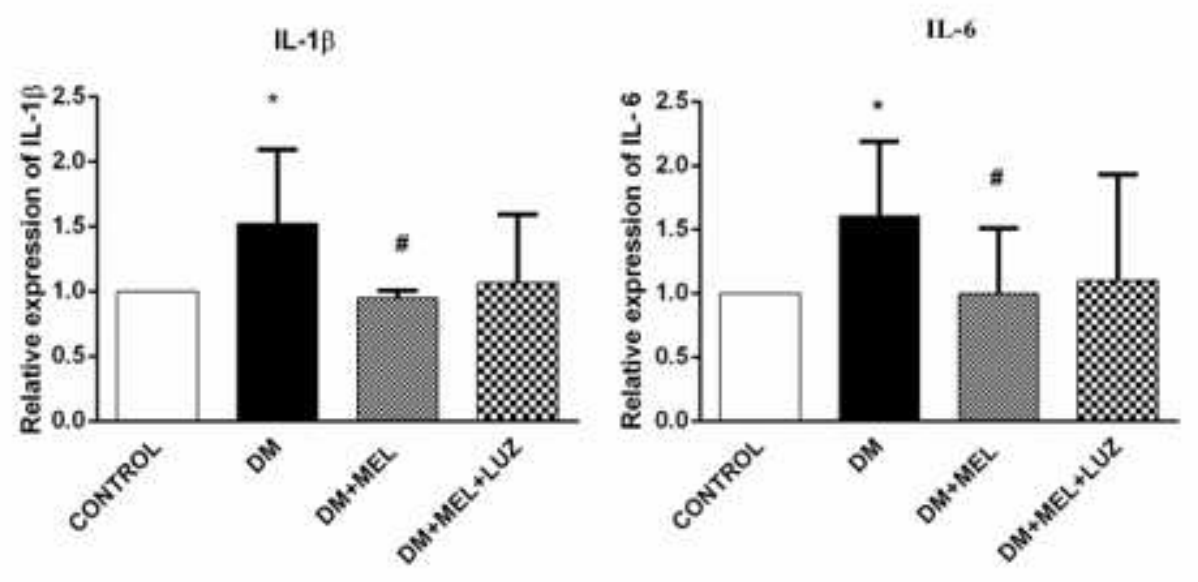

C

D
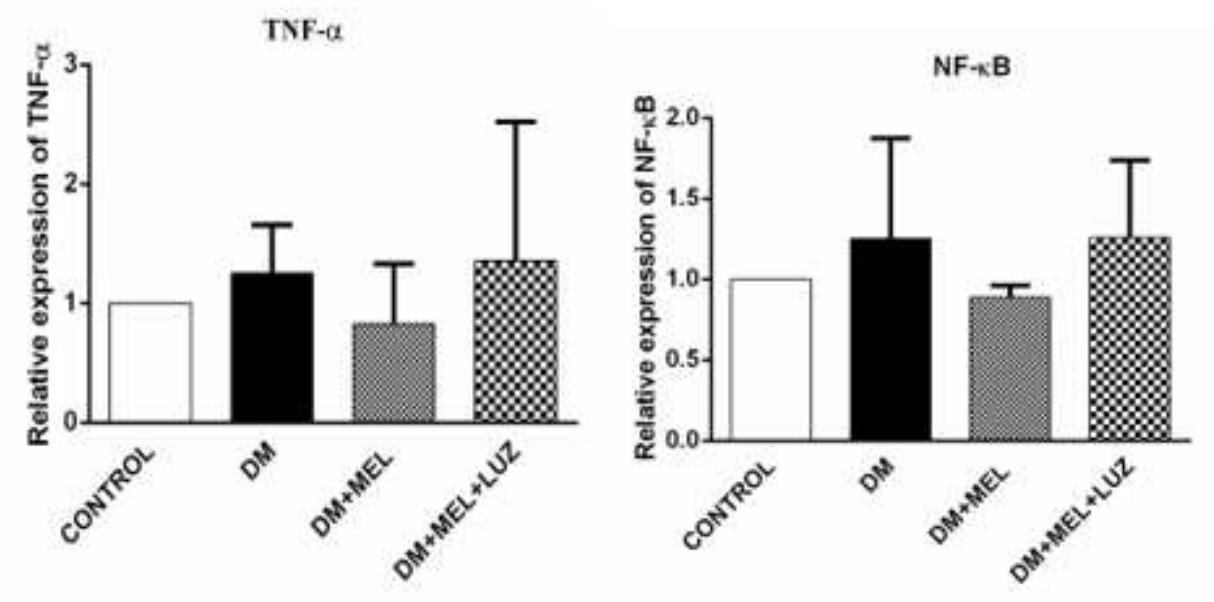

Figure 4

Q-PCR analysis results of cytokine levels in brain tissue. IL-1 3 (A) , IL-6 (B), TNF-a (C), NF-KB (D) levels in brain tissues of control, DM, DM plus treatment with melatonin $(500 \mu \mathrm{g} / \mathrm{kg} /$ day), DM plus treatment with luzindole $(0.25 \mathrm{~g} / \mathrm{kg} / \mathrm{day})$ and melatonin $(500 \mu \mathrm{g} / \mathrm{kg} /$ day $)$. Values are represented as mean $\pm S D$.

Significant differences compared to controls $=$ * Significant differences compared to $D M=\# *$ * $\#=0<0.05$ 

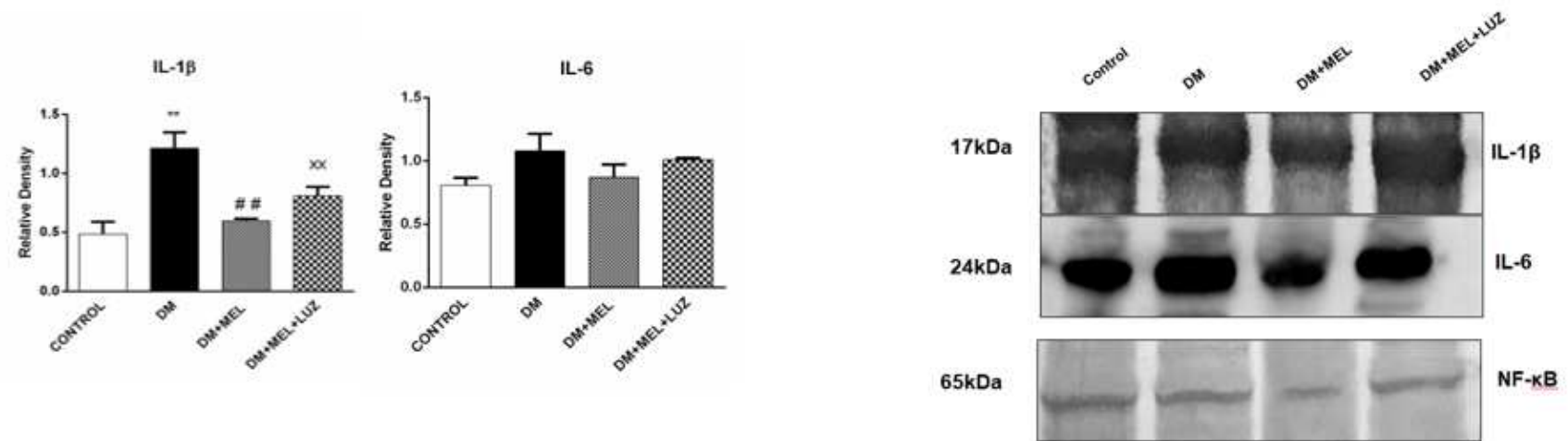

C

$$
\text { D }
$$
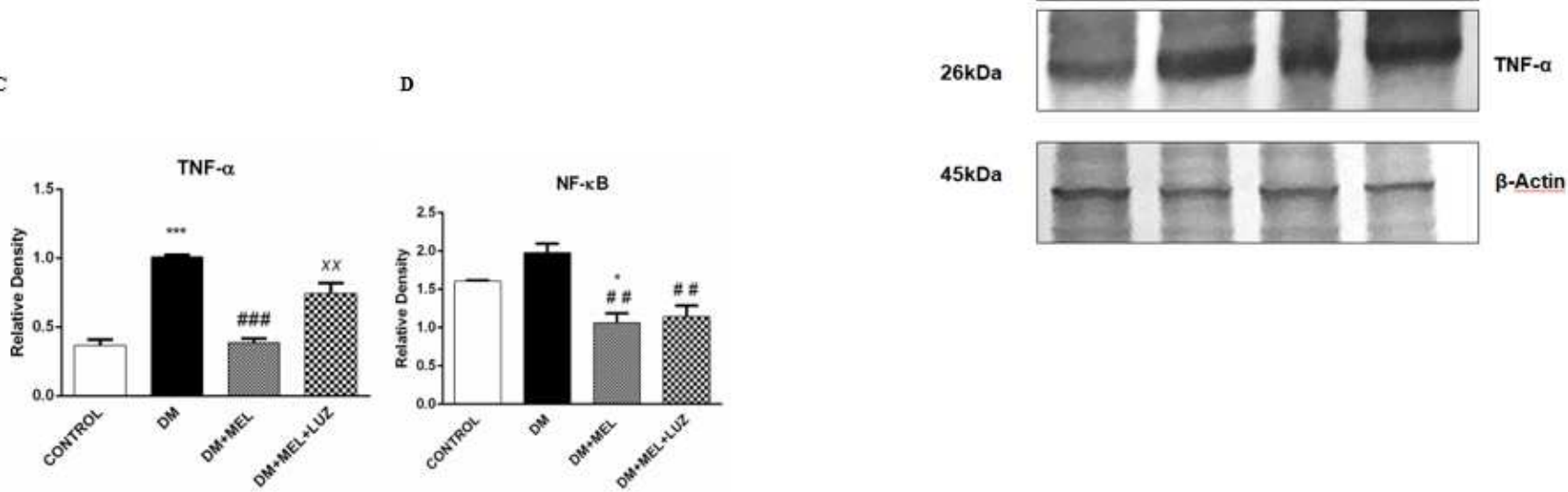

\section{Figure 5}

Western blot analysis results of cytokine levels in liver tissue. IL-1 $\beta$ (A) , IL-6 (B), TNF- $a(C), N F-K B(D)$ levels in liver tissues of control, DM, DM plus treatment with melatonin $(500 \mu \mathrm{g} / \mathrm{kg} / \mathrm{day}), \mathrm{DM}$ plus treatment with luzindole $(0.25 \mathrm{~g} / \mathrm{kg} /$ day) and melatonin $(500 \mu \mathrm{g} / \mathrm{kg} /$ day $)$. Values are represented as mean $\pm S E M$. Significant differences compared to controls $=$ * Significant differences compared to DM $=$ \# Significant differences compared to DM+MEL $=x *, \#, x=p<0.05, \star \star, \# \#, x x=p<0.01, \star \star \star, \# \# \#, x x x=$ $p<0.001$ 


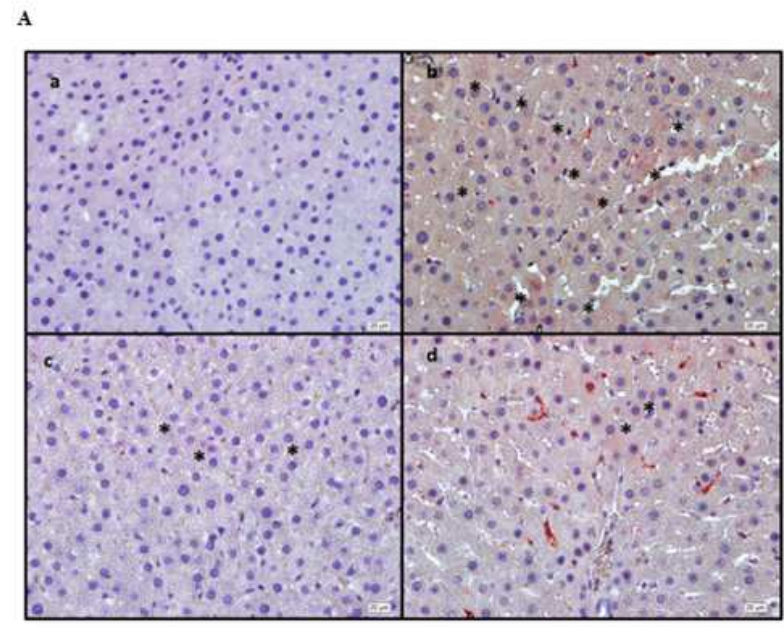

A. IL-15

a-Control; b-DM; c-DM+MEL; d-DM+MEL+LUA

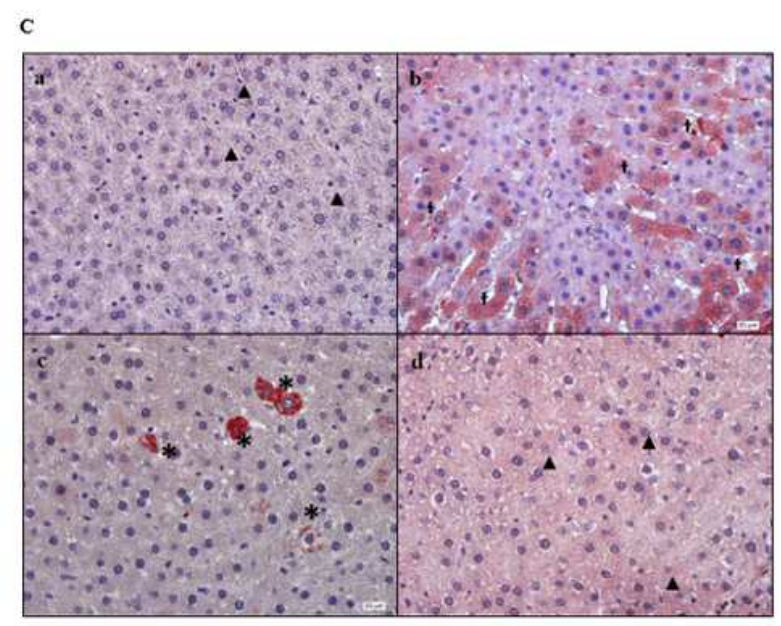

c. TNF- $a$

a-Control; b-DM; c-DM+MEL; d-DM+MEL+LUZ

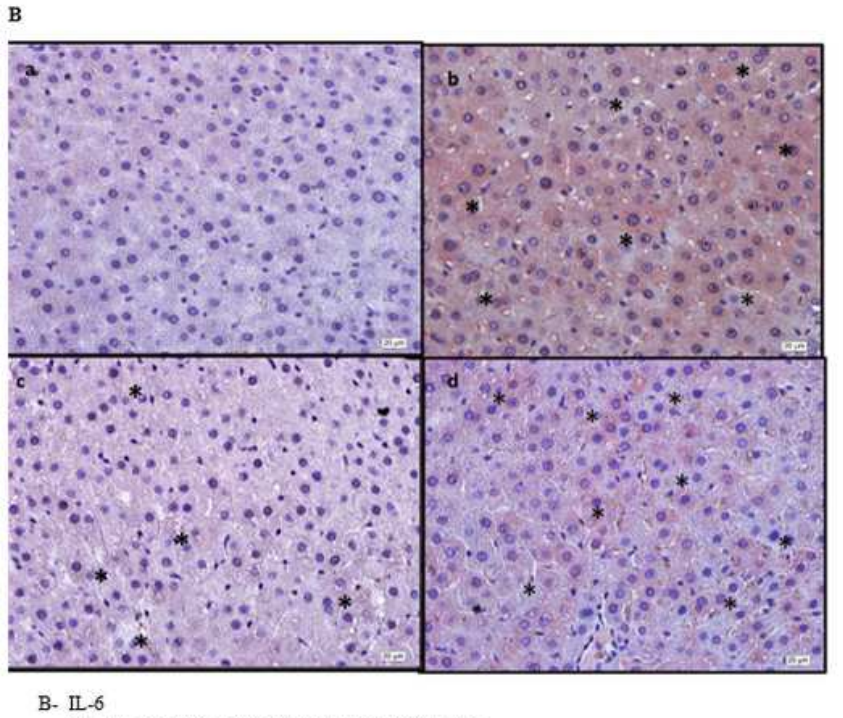

a-Control; b-DM; c-DM+MEL; d-DM+MEL +LUZ

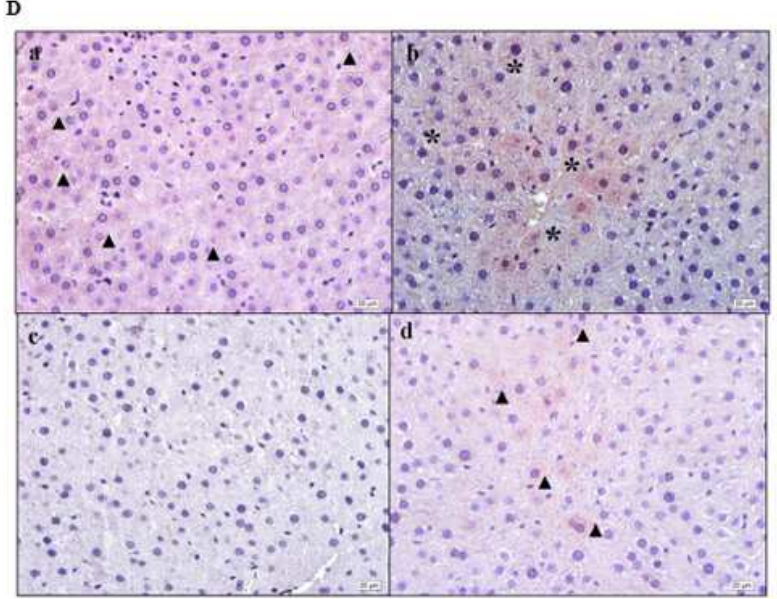

D. $\mathrm{NF}-\mathrm{KB}$

a-Control; b-DM; c-DM+MEL; d-DM+MEL+LUZ

\section{Figure 6}

Figure (6A) Representative microscobic pictures of IL-1 $\beta$ immunoreactivity of liver cross section in all experimental groups. (a) Control group: a weak immunoreactivity is observed. (b) Diabetes group: the immunoreactivity pattern is strong and different, diffused in hepatocytes cytoplasme ${ }^{*}$ ). (c) DM+MEL group: the immunoreactivity observed in cytoplasm of some cells only ( $\nabla)$. (d) Luz group: immunoreativity is moderate level in hepatocyte plate, and the signal patern is observed at cytoplasmic level (black circle). Figure (6B) Representative microscopic pictures of IL-6 immunoreactivity of liver cross-sections of all experimental groups. (a) .Control group: IL-6 immunreactivity in the cytoplasm of hepatocytes is negative. (b) DM group IL-6 immunoreactivity observed in enlarged hepatocyte cytoplasms and in heptocytes plaques $\left({ }^{\star}\right)$. (c) DM+MEL group: the space between the liver sinusoids and hepatocytes is expanded (or areas between neighbouring hepatocytes enlarged) and IL-6 immunreactivity in hepatocytes is rarely observed as intracellular dots(*). (d) Luzindole group IL-6 immunreactivity increased significantly $\left(^{*}\right)$. 
Figure (6C) Representative microscobic pictures TNF-a immunoreactivity of sections liver in all experimental groups. (a) Control group:a weak TNF-a immunoreactivity observed in hepatocyte plate branches.. (b) DM group a significantly increased immunreactivity is observed along hepatocyte plate (ه). (c) DM+MEL group: TNF-a immunreactivity observed in moderate level and strong immunreactivity observed in a few single hepatocte. (d) Luzindol group: the immunoreactivity showed a weak limited in single cell patern. Figure (6D) Representative microscobic pictures of NF-KB immunoreactivity of cross sections from liver in all experimental groups. (a) Control group: cytoplasmic pattern immunreactivity is moderate (『). (b) Diabetes group: increased immunoreactivity of NF-KB observed in hepatocyte plate $\left.{ }^{*}\right)$. (c) DM+MEL group: a negative immunoreaction patern was observed. (d) Luzindole group moderate cytoplasmic diffuse immunoreactivity pattern was observed in hepatocytes plates.

\section{Supplementary Files}

This is a list of supplementary files associated with this preprint. Click to download.

- v1.SupplementFig.Legends.docx

- SupplamentFig1lHCBrain.docx

- SupplementFig2WBBrain.docx 